\title{
METODOLOGIA PARA OBTENÇÃO DE PARÂMETROS DE MECÂNICA MOLECULAR APLICADOS A COMPOSTOS DE COORDENAÇÃ̃O
}

Carlos Henrique Ferreira Almeida, Juan Omar Machuca-Herrera e Sérgio de Paula Machado *

Departamento de Química Inorgânica, Instituto de Química, Universidade Federal do Rio de Janeiro, Cidade Universitária, CP 68563, 21945-970 Rio de Janeiro - RJ

Recebido em 3/9/01; aceito em 27/2/02

\begin{abstract}
METHODOLOGY TO OBTAIN MOLECULAR MECHANICS PARAMETERS APPLIED TO COORDINATION COMPOUNDS. A methodology is presented to obtain force field parameters to be used in molecular mechanics. The case of $\mathrm{Ru}$ (II) is investigated and the parameters obtained, specially its covalent radii, are employed to model $\mathrm{Ru}$ (II) coordination compound. The combined use of molecular mechanics with $a b$ initio methods allowed us to predict the metal-ligand stretching force constant for $\mathrm{Ru}(\mathrm{II})$ coordination compounds.
\end{abstract}

Keywords: molecular mechanics; force constant; coordination compounds.

\section{INTRODUÇÃO}

A crescente aplicação de mecânica molecular no estudo de compostos de coordenação vem sendo recentemente discutida na literatura ${ }^{1-3}$. Entretanto, é importante ressaltar que em função da complexidade apresentada por compostos envolvendo metais de transição, ainda se encontram algumas dificuldades na aplicação desta metodologia no estudo destes sistemas químicos. Esta complexidade está diretamente relacionada à descrição dos metais de transição, onde se observa determinadas características inerentes à participação dos orbitais $d$ do centro metálico. Estes fatores exercem grande influência na química de coordenação e devem ser considerados no momento em que se deseja realizar um estudo mais detalhado nesses tipos de compostos. De uma forma mais objetiva, estes fatores podem ser descritos como sendo:

a) a diversidade de números de coordenação que um mesmo metal pode apresentar;

b) os diferentes estados de oxidação existentes para o mesmo metal;

c) a capacidade que o metal possui de realizar retrodoação para o ligante;

d) a grande variedade de possíveis geometrias;

e) a existência de diferentes estados de spin.

Apesar destas peculiaridades, uma grande variedade de campos de força foram desenvolvidos e são amplamente utilizados nos cálculos de mecânica molecular, aplicados no estudo dos compostos de coordenação ${ }^{4-21}$. Alguns destes campos de força são utilizados em vários programas disponíveis comercialmente, entre os quais podemos citar: Molmec ${ }^{22}$; Momec ${ }^{23}$; Sybyl ${ }^{24}$; Shapes ${ }^{25}$; Dreiding ${ }^{26}$ e UFF $^{27}$.

Mesmo com a existência de diversos campos de força, o uso de mecânica molecular no estudo da química de coordenação ainda necessita de uma generalização, uma vez que é desejável a transferibilidade dos parâmetros adotados no campo de força.

De uma maneira mais sucinta, os principais obstáculos encontrados no uso da mecânica molecular no estudo destes sistemas químicos, são:

\footnotetext{
*e-mail: sergiopm@iq.ufrj.br
}

a) a previsão da constante de força metal-ligante, de uma forma genérica, que conserve as características químicas específicas do metal, e do ligante envolvido na ligação.

b) a modelagem mais correta da ligação química existente entre o metal e o ligante ${ }^{30}$.

Uma melhor modelagem do comprimento de ligação pode ser obtida através da utilização de parâmetros atômicos específicos de cada elemento (ex: raio covalente da espécie metálica), ao contrário dos tradicionais parâmetros por ligação específica, tornando assim, o campo de força adotado mais genérico e abrangente, sem perder a sua adaptabilidade.

Ao contrário dos elementos representativos, onde se observa uma tendência de seguir a regra clássica do octeto, os compostos de coordenação são mais complexos do ponto de vista da ligação química, tornando necessária, uma análise mais detalhada desse comportamento.

As teorias usadas até hoje para explicar qualitativamente a ligação química, em compostos de coordenação, são: a teoria de ligação de valência ${ }^{29}$, a teoria do campo cristalino ${ }^{30}$ e a teoria do orbital molecular $^{31}$. A consideração das abordagens dessas teorias torna-se de fundamental importância, uma vez que a interação metal-ligante é um dos fatores que dificultam o estabelecimento de um campo de força generalizado. Desta forma, conclui-se que para o estudo mais detalhado dos compostos de coordenação, utilizando mecânica molecular, é importante incluir fatores que possam descrever melhor as interações covalentes e iônicas, existentes na ligação química entre o metal e o ligante.

A introdução destes termos pode ser realizada através do acoplamento dos fatores iônico e covalente nas equações que descrevem o comprimento de ligação, uma vez que, ao incorporar estes dois termos na estimativa do comprimento de ligação, estes estariam diretamente inseridos no tratamento da constante de força de estiramento metal-ligante.

\section{METODOLOGIA}

Todos os cálculos ab initio realizados neste trabalho, foram feitos em um computador PC Pentium II $450 \mathrm{MHz}$, utilizando o programa de orbital molecular GAMESS ${ }^{32}$. Os cálculos de mecânica 
molecular foram realizados em um computador PC por meio do programa FORCES ${ }^{33}$ desenvolvido em nosso laboratório.

\section{RESULTADOS E DISCUSSÃO}

\section{Previsão do comprimento da ligação metal-ligante}

Uma das primeiras expressões propostas para determinar o comprimento de ligação entre dois átomos $\left(\mathrm{D}_{\mathrm{ML}}\right)$, considerando o fator iônico e o fator covalente em uma ligação química, foi desenvolvida por Schomaker e colaboradores ${ }^{34}$, que descreveram o comprimento de ligação entre dois átomos, através da seguinte equação:

$\mathrm{D}_{\mathrm{ML}}=\mathrm{r}_{\mathrm{M}}+\mathrm{r}_{\mathrm{L}}-0,09 *\left(\chi_{\mathrm{M}}-\chi_{\mathrm{L}}\right)$

Na equação 1 tem-se os valores de raio covalente do metal $\left(r_{M}\right)$, raio covalente do ligante $\left(r_{L}\right)$, a eletronegatividade do metal $\left(\chi_{M}\right)$ e do ligante $\left(\chi_{\mathrm{L}}\right)$, além de uma constante empírica de proporcionalidade igual a 0,09 .

Nesta equação observa-se a contribuição de um caráter iônico, representado pelas diferenças de eletronegatividade, e de um caráter covalente, representado pelo uso dos valores de raios covalentes.

Uma outra forma de prever o comprimento de ligação entre dois átomos, e de uma forma mais específica entre um íon metálico e seu ligante, foi desenvolvida por See e colaboradores ${ }^{35}$, introduzindo o conceito de ordem de ligação na previsão da distância entre dois átomos.

$\mathrm{D}_{\mathrm{ML}}=\mathrm{R}_{\mathrm{ML}}-0,37 \ln \left(\mathrm{OL} / \mathrm{n}^{\circ} \mathrm{de}\right.$ coordenação $)$

Na equação 2 tem-se agora a soma dos valores de raio iônico do metal e do ligante $\left(\mathrm{R}_{\mathrm{ML}}\right)$, a ordem de ligação (OL), além da constante empírica de proporcionalidade igual a 0,37. Nesta equação, o caráter iônico está representado pela soma dos raios iônicos e o fator covalente pela ordem de ligação. Os valores de raios iônicos dos metais de transição, assim como dos elementos representativos, são descritos na literatura por Shannon-Prewitt ${ }^{36}$, que faz a determinação destes valores em função da carga e do número de coordenação existente no composto.

Uma terceira equação utilizada para a previsão do comprimento de ligação, que é usada no campo de força do programa de mecânica molecular $\mathrm{UFF}^{27}$, foi desenvolvida por Goddard III a partir da equação de Shomaker (equação 1), e descreve o comprimento de ligação entre dois elementos, como sendo:

$\mathrm{D}_{\mathrm{ML}}=\mathrm{r}_{\mathrm{M}}+\mathrm{r}_{\mathrm{L}}+\mathrm{r}_{\mathrm{OL}}+\mathrm{r}_{\mathrm{EN}}$

Na equação 3, assim como na equação 1 , a contribuição iônica da ligação está representada pelo fator de correção da eletronegatividade $\left(\mathrm{r}_{\mathrm{EN}}\right)$, enquanto que a contribuição covalente está representada pelos raios covalentes do íon metálico e do ligante. Existe também na equação 3 o fator de correção por ordem de ligação $\left(\mathrm{r}_{\mathrm{OL}}\right)$, que tem por objetivo descrever as contribuições das ligações múltiplas, podendo-se afirmar que sem a inclusão dessa correção, a ligação química apresentaria apenas caráter de ligação simples.

Desta maneira a correção de ordem de ligação tem um importante papel na descrição da interação metal-ligante, visto que nem sempre a ligação química existente em compostos de coordenação terá caráter simples devido, entre outros fatores, à possibilidade da ocorrência de retrodoação $\pi$ entre o íon metálico e o ligante.

No estudo dos compostos de coordenação, uma outra razão para a inclusão da ordem de ligação na descrição da ligação química devese ao fato dos elementos envolvidos apresentarem diferentes estados de oxidação. Entretanto, os valores de eletronegatividade para o metal, utilizados dentro da correção de eletronegatividade $\left(\mathrm{r}_{\mathrm{EN}}\right)$ na equação 3, são valores obtidos para o estado de oxidação zero. Desta forma, através da correção de ordem de ligação, a natureza do átomo envolvido na ligação química, será melhor descrita, minimizando a diferença entre os estados de oxidação.

$\mathrm{O}$ fator de correção de eletronegatividade $\left(\mathrm{r}_{\mathrm{EN}}\right)$ utilizado na equação 3 foi desenvolvido por O'Keefe e Brese ${ }^{37}$, e é descrito pela equação 4:

$r_{E N}=\frac{r_{M} * r_{L}\left(\chi_{M}-\chi_{L}\right)^{2}}{\chi_{M} * r_{M}+\chi_{L} * r_{L}}$

$\mathrm{O}$ fator de correção da ordem de ligação $\left(\mathrm{r}_{\mathrm{OL}}\right)$ discutido anteriormente, foi desenvolvido por Pauling ${ }^{29}$, e é descrito pela equação 5:

$\mathrm{r}_{\mathrm{OL}}=-0,1332\left(\mathrm{r}_{\mathrm{M}}+\mathrm{r}_{\mathrm{L}}\right) \ln \mathrm{OL}$

E a ordem de ligação OL pode ser descrita de acordo com a equação $6^{33}$ :

$\mathrm{OL}=\exp \left[\left(\mathrm{R}_{\mathrm{ML}}-\mathrm{D}_{\mathrm{ML}}\right) / 0,37\right]$

$\mathrm{Na}$ equação 5 tem-se uma nova constante empírica de proporcionalidade igual a 0,1332 , enquanto que na equação $6, \mathrm{R}_{\mathrm{ML}}$ agora é dada em função da soma dos raios covalentes do metal e do ligante, e $\mathrm{D}_{\mathrm{ML}}$ é a distância real de ligação.

As equações descritas indicam que a descrição do comprimento de ligação entre o metal e o ligante pode ser feita pela aplicação de parâmetros atômicos, ou seja, característicos de cada elemento envolvido na ligação, conferindo um maior grau de fidelidade na descricão das ligações químicas a serem estudadas.

O suporte para a aplicação destas equações deve-se à grande parte dos parâmetros necessários para a previsão do comprimento de ligação metal-ligante estarem disponíveis na literatura, tais como valores de raios iônicos e de eletronegatividade, tanto para o metal como para o ligante, facilitando, desta maneira, a implementação destas equações dentro do campo de força adotado.

\section{CÁLCULO DA CONSTANTE DE FORÇA METAL-LIGANTE}

A associação do comprimento de ligação entre o íon metálico e o ligante com a constante de força de estiramento metal-ligante torna o tratamento desta constante mais confiável, uma vez que tanto o fator iônico quanto o fator covalente, existentes nos compostos de coordenação, estarão incorporados na previsão do comprimento de ligação, conforme discutido anteriormente. Desta forma, estes fatores estarão diretamente inseridos no valor da constante de força.

Existem diversas equações que relacionam a constante de força de estiramento com o comprimento de ligação, destacando-se as seguintes equações:

a) Equação de Badger $^{38}$ :

$K=\frac{A}{\left(D_{M L}-B\right)^{3}}$

b) Equação em termos de ordem de ligação ${ }^{39}$ :

$\mathrm{K}=1,67 * \mathrm{~N} *\left(\chi_{\mathrm{M}} * \chi_{\mathrm{L}} / \mathrm{D}^{2}{ }_{\mathrm{ML}}\right)^{0,75}+0,3$

Como pode ser observado, a equação 7 possui duas constantes relacionadas à natureza dos átomos ligados (A e B), enquanto que 
na equação 8 existem outras constantes empíricas de proporcionalidade de valores 1,67 e 0,3 .

No presente trabalho ${ }^{40}$, realizou-se o estudo de um complexo octaédrico de rutênio(II), por meio de mecânica molecular, através da parametrização de um campo de força, obtida a partir de resultados de cálculos de orbital molecular realizados a nível ab initio.

$\mathrm{O}$ uso conjunto das metodologias de mecânica molecular e de orbital molecular vem se tornando cada vez mais comum, conforme relatam vários trabalhos na literatura, que demonstram a validade desta metodologia ${ }^{41-44}$.

A primeira etapa do trabalho consistiu na previsão do valor de raio covalente da espécie $\mathrm{Ru}(\mathrm{II})$. Embora estejam disponíveis na literatura os parâmetros atômicos utilizados na equação 3, existe uma grande dificuldade na determinação de parâmetros relacionados ao raio covalente do metal em seus diversos estados de oxidação.

Para a determinação do raio covalente $\left(r_{M}\right)$, aplicou-se a equação 3 em uma série de complexos de rutênio II, onde se conhecem as distâncias interatômicas medidas experimentalmente por difração de raios- $\mathrm{X}^{45,46}$. Na realização destes cálculos, além dos comprimentos de ligação experimentais, utilizou-se a escala de eletronegatividade de Allred-Rochow ${ }^{47}$ e os parâmetros âtomicos calculados por Allinger ${ }^{39}$ para os ligantes envolvidos. Os valores obtidos, utilizando esta metodologia, são apresentados nas Tabelas 1,2 e 3 .

Tabela 1. Distâncias interatômicas ${ }^{43}$ e respectivos valores do raio covalente do íon metálico, calculado através da equação 3 no complexo $\mathrm{RuCl}_{2}(\mathrm{CO})_{2}\left(\mathrm{PPh}_{3}\right)_{2}$

\begin{tabular}{lcc}
\hline Ligação & Distância $(\AA)$ & $r_{M}(\AA)$ \\
\hline $\mathrm{Ru}-\mathrm{Cl}$ & 2,4260 & 1,1607 \\
$\mathrm{Ru}-\mathrm{Cl}$ & 2,4530 & 1,1775 \\
$\mathrm{Ru}-\mathrm{C}$ & 1,8650 & 1,2258 \\
$\mathrm{Ru}-\mathrm{C}$ & 1,8540 & 1,2153 \\
$\mathrm{Ru}-\mathrm{P}$ & 2,4247 & 1,2076 \\
$\mathrm{Ru}-\mathrm{P}$ & 2,4249 & 1,2078 \\
\hline
\end{tabular}

Tabela 2. Distâncias interatômicas ${ }^{43}$ e respectivos valores do raio covalente do íon metálico, calculado através da equação 3 no complexo $\mathrm{RuCl}_{2}(\mathrm{CO})_{2}\left(\mathrm{AsPh}_{3}\right)_{2}$

\begin{tabular}{lcc}
\hline Ligação & Distância $(\AA)$ & $r_{M}(\AA)$ \\
\hline $\mathrm{Ru}-\mathrm{Cl}$ & 2,426 & 1,1607 \\
$\mathrm{Ru}-\mathrm{Cl}$ & 2,443 & 1,1713 \\
$\mathrm{Ru}-\mathrm{C}$ & 1,873 & 1,2335 \\
$\mathrm{Ru}-\mathrm{C}$ & 1,848 & 1,2096 \\
\hline
\end{tabular}

Tabela 3. Distâncias interatômicas ${ }^{44}$ e respectivos valores do raio covalente do íon metálico, calculado através da equação 3 no complexo $\mathrm{RuCl}_{2}(\mathrm{CO})_{2}\left(\mathrm{BzlPPh}_{2}\right)_{2}$

\begin{tabular}{lcc}
\hline Ligação & Distância $(\AA)$ & $r_{M}(\AA)$ \\
\hline $\mathrm{Ru}-\mathrm{Cl}$ & 2,430 & 1,1632 \\
$\mathrm{Ru}-\mathrm{Cl}$ & 2,428 & 1,1619 \\
$\mathrm{Ru}-\mathrm{C}$ & 1,869 & 1,2297 \\
$\mathrm{Ru}-\mathrm{C}$ & 1,858 & 1,2192 \\
$\mathrm{Ru}-\mathrm{P}$ & 2,410 & 1,1963 \\
$\mathrm{Ru}-\mathrm{P}$ & 2,410 & 1,1963 \\
\hline
\end{tabular}

Comparando os resultados obtidos para o raio covalente do íon $\mathrm{Ru}(\mathrm{II})$ com o valor existente na literatura ${ }^{37}$, de aproximadamente $1,24 \AA$ A para o rutênio metálico, pode-se observar que os valores de raio covalente obtidos para $\mathrm{Ru}(\mathrm{II})$ usando a eletronegatividade de Allred-Rochow, apresentaram resultados numericamente menores que o valor de raio covalente usado por Allinger sendo, portanto, coerentes sob o ponto de vista químico. Desta maneira, atribuiu-se para o raio covalente da espécie $\mathrm{Ru}(\mathrm{II})$ o valor de $1,19 \AA$ que é a média aritmética dos valores calculados e apresentados nas Tabelas 1,2 e 3.

Paralelamente à determinação do raio covalente da espécie $\mathrm{Ru}^{\mathrm{II}}$, utilizou-se o programa de orbital molecular GAMESS na realização de cálculos ab initio a nível Hartree-Fock usando as bases MIDI e o potencial efetivo ECP-SBK, no intuito de estimar a constante de força de estiramento da ligação $\mathrm{Ru}^{\mathrm{II}}-\mathrm{N}$.

Nesta etapa é importante ressaltar que não foram incorporados efeitos de correlação eletrônica nos cálculos de orbital molecular, como por exemplo, cálculos a nível MP2, devido ao fato dos modelos adotados possuirem um metal da segunda série de transição, o que tornaria o custo computacional deste tipo de cálculo extremamente elevado.

Inicialmente otimizou-se a geometria de complexos modelo que contém o mesmo íon central $\left(\mathrm{Ru}^{\mathrm{II}}\right)$ e o átomo de nitrogênio como ligante, utilizando simetria $\mathrm{Cs}$, a fim de diminuir o esforço computacional envolvido. As estruturas dos complexos modelo estão dispostas na Figura 1.<smiles>O=N[R](Cl)(Cl)(Cl)(Cl)Cl</smiles>

$\left[\mathrm{RuCl}_{5} \mathrm{NO}\right]^{2-}$

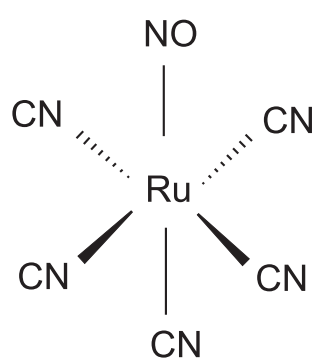

$\left[\mathrm{Ru}(\mathrm{CN})_{5} \mathrm{NO}\right]^{2-}$
Figura 1. Ânions complexos de Ru(II) modelados pelo método ab initio

Após a otimização de geometria, aplicou-se os valores obtidos de comprimento da ligação $\mathrm{Ru}^{\mathrm{II}}-\mathrm{N}$, além das respectivas ordem de ligação, na equação de Badger (equação 7) e na equação de ordem de ligação (equação 8), e determinou-se o valor da constante de força de estiramento da ligação $\mathrm{Ru}^{\mathrm{II}}-\mathrm{N}$. Os valores de eletronegatividade usados na equação 8 também foram obtidos da escala de AllredRochow.

Os valores das constantes de força calculadas pelas equações $7 \mathrm{e}$ 8 , e da constante calculada pelo próprio método $a b$ initio encontram-se na Tabela 4, onde são comparados com um valor de constante de força $\mathrm{Ru}^{\mathrm{II}}-\mathrm{N}$ encontrado na literatura ${ }^{48}$.

Através da análise da Tabela 4, observou-se uma boa concordância entre o valor encontrado na literatura, e o valor calculado utilizando a equação de ordem de ligação (equação 8) com a base MIDI. Em relação às constantes de força determinadas pelo uso de cálculos $a b$ initio, observa-se uma tendência já destacada na literatura ${ }^{49}$, que é a obtenção de valores superestimados, quando comparados com os valores experimentais.

$\mathrm{Na}$ análise dos resultados das constantes de força, calculadas por intermédio da equação de Badger (equação 7), podemos observar 
Tabela 4. Constantes de força (mydn/Å) do estiramento Ru-N

\begin{tabular}{|c|c|c|c|c|}
\hline & $\mathrm{MIDI}\left[\mathrm{Ru}(\mathrm{CN})_{5} \mathrm{NO}\right]^{2-}$ & $\mathrm{SBK}\left[\mathrm{Ru}(\mathrm{CN})_{5} \mathrm{NO}\right]^{2-}$ & $\mathrm{MIDI}\left[\mathrm{RuCl}_{5} \mathrm{NO}\right]^{2-}$ & $\mathrm{SBK}\left[\mathrm{RuCl}_{5} \mathrm{NO}^{2-}\right.$ \\
\hline Equação 8 & 2,6645 & 3,3219 & 2,6362 & 2,8549 \\
\hline Equação 7 & 4,6610 & 4,5941 & 4,7280 & 4,6907 \\
\hline GAMESS & 7,2809 & 6,5949 & 7,0590 & 5,9969 \\
\hline Literatura $^{47}$ & & 2,6837 & & \\
\hline
\end{tabular}

que os resultados obtidos estão distantes do valor experimental. Este fato pode ser justificado pelo fato dos valores das constantes A e B da equação 7, encontrados para os elementos envolvidos na ligação, não estarem bem descritos para o sistema em questão, neste caso, contendo o íon $\mathrm{Ru}(\mathrm{II})^{50}$. Desta forma, aferiu-se como valor da constante de força de estiramento $\mathrm{Ru}^{\mathrm{II}}-\mathrm{N}$, o resultado obtido pela equação de ordem de ligação (equação 8) com os dados calculados pela base MIDI.

Com o estabelecimento da constante de força de 2,66 mydn/Å, aplicou-se este valor na modelagem molecular do complexo $\mathrm{RuCl}_{2} \mathrm{~L}(\mathrm{CO})\left(\mathrm{PPh}_{3}\right)_{2}(\mathrm{~L}=4$-picolina $)$, uma vez que este composto apresenta a espécie Ru(II) como íon central, além de possuir um ligante que se coordena ao metal através do átomo de nitrogênio.

Para isso, utilizou-se o programa de mecânica molecular FORCES, que utiliza o campo de força de valência geral, para testar o valor da constante estabelecida, e do raio covalente da espécie $\mathrm{Ru}(\mathrm{II})$ previsto anteriormente, através de um cálculo de otimização de geometria.

A estrutura do complexo em sua geometria otimizada está ilustrada na Figura 2.

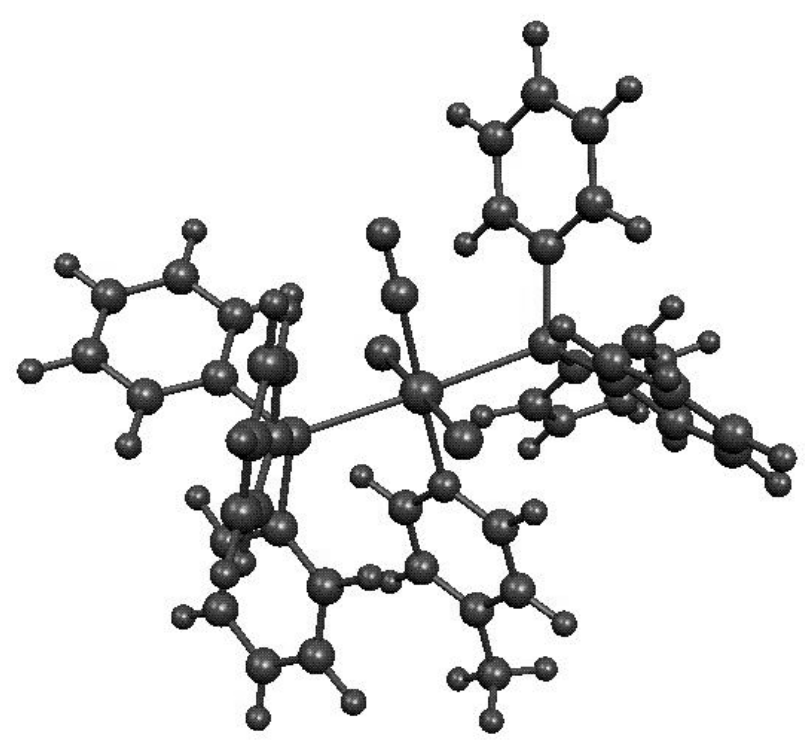

Figura 2. Estrutura otimizada do composto $\mathrm{RuCl}_{2} \mathrm{~L}(\mathrm{CO})\left(\mathrm{PPh}_{3}\right)_{2} \mathrm{~L}=4$ picolina

A análise estrutural do composto obtida, assim como a comparação com os valores experimentais obtidos por difração de raios- $X^{51}$, são mostrados nas Tabelas 5 e 6 .

Através da análise destes resultados, podemos observar uma excelente concordância entre os valores calculados e experimentais tanto em relação ao comprimento de ligação, como em relação aos ângulos envolvendo o átomo central.
Tabela 5. Valores médios das distâncias interatômicas do complexo $\mathrm{RuCl}_{2} \mathrm{~L}(\mathrm{CO})\left(\mathrm{PPh}_{3}\right)_{2} \quad \mathrm{~L}=4$-picolina

\begin{tabular}{lcc}
\hline Ligação & Experimental $(\AA)^{51}$ & Calculado $(\AA)$ \\
\hline $\mathrm{Ru}-\mathrm{P}$ & 2,4050 & 2,5350 \\
$\mathrm{Ru}-\mathrm{C}$ & 2,0100 & 1,9530 \\
$\mathrm{Ru}-\mathrm{Cl}$ & 2,4170 & 2,3340 \\
$\mathrm{Ru}-\mathrm{N}$ & 2,2020 & 1,9660 \\
$\mathrm{C}-\mathrm{O}$ & 1,1000 & 1,1630 \\
\hline
\end{tabular}

Tabela 6. Ângulos em graus envolvendo o íon $\mathrm{Ru}^{2+}$ e ligantes do complexo $\mathrm{RuCl}_{2} \mathrm{~L}(\mathrm{CO})_{2}\left(\mathrm{PPh}_{3}\right) \mathrm{L}=4$-picolina

\begin{tabular}{lcc}
\hline Ângulo & Experimental $^{51}$ & Calculado \\
\hline $\mathrm{P}-\mathrm{Ru}-\mathrm{P}$ & 175,50 & 176,19 \\
$\mathrm{C}-\mathrm{Ru}-\mathrm{N}$ & 175,50 & 178,97 \\
$\mathrm{Cl}-\mathrm{Ru}-\mathrm{Cl}$ & 178,80 & 178,35 \\
$\mathrm{P}-\mathrm{Ru}-\mathrm{Cl}$ & 90,60 & 91,92 \\
$\mathrm{P}-\mathrm{Ru}-\mathrm{Cl}$ & 93,10 & 89,95 \\
$\mathrm{C}-\mathrm{Ru}-\mathrm{Cl}$ & 87,30 & 90,83 \\
$\mathrm{Cl}-\mathrm{Ru}-\mathrm{N}$ & 89,60 & 89,20 \\
$\mathrm{P}-\mathrm{Ru}-\mathrm{Cl}$ & 88,50 & 87,17 \\
$\mathrm{P}-\mathrm{Ru}-\mathrm{Cl}$ & 87,80 & 91,02 \\
$\mathrm{C}-\mathrm{Ru}-\mathrm{Cl}$ & 93,50 & 90,53 \\
$\mathrm{~N}-\mathrm{Ru}-\mathrm{Cl}$ & 89,50 & 89,44 \\
$\mathrm{P}-\mathrm{Ru}-\mathrm{C}$ & 94,30 & 89,12 \\
$\mathrm{P}-\mathrm{Ru}-\mathrm{N}$ & 89,00 & 91,90 \\
$\mathrm{C}-\mathrm{Ru}-\mathrm{P}$ & 88,40 & 87,53 \\
$\mathrm{P}-\mathrm{Ru}-\mathrm{N}$ & 88,50 & 91,43 \\
\hline
\end{tabular}

As diferenças estruturais observadas nos resultados são devidas principalmente ao fator de empacotamento cristalino existente na estrutura em seu estado sólido, que não permite o mesmo grau de liberdade que este composto possuiria no estado gasoso, que é o estado em que a molécula é considerada num processo de modelagem molecular.

\section{CONCLUSÃO}

A utilização de uma equação que possa determinar o comprimento de ligação, considerando tanto o fator covalente quanto o fator iônico, com a aplicação de valores de constante de força de estiramento baseados em resultados a nível $a b$ initio, é uma ferramenta fundamental no estudo de compostos de coordenação por mecânica molecular. Além de tornar possível a análise das propriedades eletrônicas dos compostos de coordenação, por meio do acoplamento da mecânica molecular com o orbital molecular (MM$\mathrm{OM})^{52-55}$. Este acoplamento é uma alternativa importante, uma vez que o estudo das propriedades eletrônicas de complexos com um 
elevado número de átomos pelo método $a b$ initio envolve um grande custo computacional.

A importância deste trabalho torna-se evidente quando a aplicação da equação 3 possibilita a estimativa de parâmetros até então inexistentes na literatura, como é o caso de valores de raios covalentes de espécies metálicas com diferentes estados de oxidação.

Esta metodologia demonstra a possibilidade de se conservar as características atômicas de cada elemento envolvido na interação metal-ligante na aplicação de mecânica molecular nos compostos de coordenação, fornecendo uma descrição melhor das ligações químicas para a modelagem destes sistemas. Este trabalho fornece, também, a metodologia para se aperfeiçoar ainda mais os campos de força já existentes para estudos mais elaborados de complexos envolvendo íons metálicos.

\section{AGRADECIMENTOS}

Os autores gostariam de agradecer à CAPES, FAPERJ e FUJB pelo apoio financeiro dado a este trabalho.

\section{REFERENCIAS}

1. Boeyens, J. C. A.; Comba, P.; Coord. Chem Rev. 2001, 212, 3

2. Deeth, R. J.; Coord. Chem Rev. 2001, 212, 11.

3. Norrby, P. O.; Brandt, P.; Coord. Chem Rev. 2001, 212, 79.

4. Burket, U.; Allinger, N.L.; Molecular Mechanics, ACS Monograph 177: Washington D.C., 1982.

5. Boyd, R. H.; J. Chem. Phys. 1968, 49, 2574.

6. Bartol, J.; Comba, P.; Melter, M.; Zimmer, M.; J. Comput. Chem. 1999, 20, 1549.

7. Comba, P.; Goll, W.; Nuber, B.; Varnagy, K.; Eur. J. Inorg. Chem. 1998, 12, 2041.

8. Da Cruz, M. F. ; Zimmer, M.; Inorg.Chem. 1998, 37, 366.

9. Hunger, J.; Beyreuther, S.; Huttner, G.; Allinger, K.; Radelof, U.; Zsolnai, L.; Eur. J. Inorg. Chem. 1998, 6, 693.

10. Hancock, R.D.; Weaving, J.S.; Marques, H.M.; J. Chem. Soc. Chem. Commun. 1989, 16, 1176.

11. Geremia, S.; Vicentini, L.; Calligaris, M.; Inorg. Chem. 1998, 37, 4094.

12. Comba, P.; Inorg. Chem. 1989, 28, 426.

13. Sabolovic, J.; Raos, N.; J. Mol. Struct. (Theochem.) 1993, 100, 101.

14. Davies, S. G.; Derome, A. E.; Mcnally, J. P.; J. Am. Chem. Soc. 1991, 113, 2854.

15. Hagelin, H.; Svensson, M.; Akermark, B.; Norrby, P. O.; Organometallics 1999, 18, 4574.

16. Zimmer, M.; Chem. Rev. 1995, 95, 2629.

17. Georgieva, I.; Trendafilova, N.; Monatsh. Chem. 1997, 128, 1119.

18. Coelho, L. W.; Junqueira, G. M. A.; Machuca-Herrera, J. O.; Machado. S. P; Machado, B. C.; Quim. Nova 1999, 22, 396.

19. Comba, P.; Coord. Chem. Rev. 1999, 123, 1.

20. Comba, P.; Coord. Chem Rev. 1999, 182, 343.

21. Hay, B. P.; Coord. Chem. Rev. 1993, 126, 177.

22. Adam, K. R.; Antolovich, M.; Bridgen, L. G.; Lindy, L. F.; J. Am. Chem. Soc. 1991, 113, 3346.
23. Comba, P.; Hambley, T.W.; Okon, N.; Momec, A Strain Energy Minimization Package Adapted to Hyperchem ${ }^{T M}$, Altenhoff \& Schmitz: Germany, 1995.

24. Hancock, R. D.; Hegetschweiler, K.; J. Chem. Soc., Dalton Trans. 1993, 2137.

25. Allured, V. S.; Kelly, C. M.; Landis, C. R.; J. Am. Chem. Soc. 1991, 113, 1.

26. Mayo, S. L.; Olafson, B. D.; Goddard III, W. A.; J. Phys. Chem. 1990, 94, 8897.

27. Rappé, A. K.; Casewit, C. J.; Colwell, K. S.; Goddard III, W. A.; J. Am. Chem. Soc. 1992, 114, 10024.

28. Comba, P.; Hambley, T.W.; Molecular Modeling of Inorganic Compounds, VCH: Weinheim, 1995.

29. Pauling, L.; The Nature Of Chemical Bond and the Structures of Molecules and Crystals: An Introduction to Modern Chemistry, Cornell University Press: New York, 1960.

30. Bethe, H.; Ann. Phys. 1929, 3, 135

31. Pople, J. A.; Schleyer, P. V. R.; Radom, L.; Hehre, W. J.; Ab Initio Molecular Orbital Theory, Wiley Interscience: New York, 1986.

32. Schmidt, M. W.; Baldridge, K. K.; Boatz, J. A.; Elbert, S. T.; Gordon, M. S.; Jensen, J. H.; Koseki, S.; Matsunaga, N.; Nguyen, K. A.; Su, S.; Windus, L.; Dupuis, M.; Montgomery, J. A.; J. Comput. Chem. 1993, 14, 1347.

33. Machuca-Herrera, J. O.; Tese de Doutorado, Universidade Estadual de Campinas, Brasil, 1988.

34. Schomaker, V.; Stevenson, D. P.; J. Am. Chem. Soc. 1941, 63, 37.

35. See, F. R.; Kruse, R. A.; Strub, W. M.; Inorg. Chem. 1998, 37, 5369.

36. Shannon, R. D.; Acta Crystallogr., Sect. A.: Found. Crystallogr. 1976, A 32,751 .

37. O'Keeffe, M.; Brese, N.E.; J. Am. Chem. Soc. 1991, 113, 3226.

38. Badger, R. M.; J. Chem. Pyhs. 1933, 2, 128.

39. Allinger, N. L.; Zhou, X.; Bergsma, J.; J. Mol. Struct. (Theochem.) 1994, $312,69$.

40. Almeida, C. H. F.; Dissertação de Mestrado, Universidade Federal do Rio de Janeiro, Brasil, 1999.

41. Norrby, P-O.; Liljefors, T.; J. Comput. Chem. 1998, 19, 1146.

42. Maseras, F.; Chem. Commun. 2001, 19, 1821.

43. Woo, T. K.; Pioda, G.; Rothlisberger, U.; Togni, A.; Organometallics 2000, 19, 2144.

44. Margl, P.; Deng, L. Q.; Ziegler, T.; Organometallics 1999, 18, 5701.

45. Batista, A. A.; Zukerman-Schpector, J.; Porcu, O. M.; Queiroz, S. L.; Araújo, M. P.; Polyhedron 1994, 13, 689.

46. Wilkes, L. M.; Nelson, J. H.; Mitchener, J. P.; Babich, M. W.; Riley, W. C.; Helland, B. J.; Jacobson, R. A.; Cheng, M. Y.; Seff, K.; Mccusker, L. B.; Inorg. Chem. 1982, 21, 1376.

47. Allred, A. L.; Rochow, EG.; J. Inorg. Nucl. Chem. 1958, 5, 264.

48. Brandt, P.; Norrby, T.; Akermark, B.; Inorg. Chem. 1998, 37, 4120.

49. Clark, T.; A Handbook Of Computational Chemistry; A Pratical Guide to Chemical Structure and Energy Calculations, John Wiley and Sons: New York, 1985.

50. Wittbrodt, J. N.; Schlegel, H. B.; J. Mol. Struct. (Theochem.) 1997, 398$399,55$.

51. Wohnrath, K.; Batista, A. A.; Ferreira, A.G.; Zukerman-Schpector, J.; Oliveira, L. A. A.; Castellano, E.E.; Polyhedron 1998, 11-12, 2013.

52. Junqueira, G. M. A.; Dissertação de Mestrado, Universidade Federal do Rio de Janeiro, Brasil, 1998.

53. Khoroshun, D. V.; Musaev, D. G.; Morokuma, K.; Organometallics 1999, $18,5653$.

54. Cummings, P. L.; Gready, J. E.; J. Comput. Chem. 1999, 20, 1028.

55. Schoemaker, J. R.; Burggraf, L. W.; Gordon, M. S.; J. Phys. Chem. A 1999, 103,3245 . 